\title{
Neurological concomitants of uveitis
}

\section{J R Smith, J T Rosenbaum}

Aim: To describe the prevalence and types of neurological disease that occur in association with uveitis.

Methods: Retrospective review of medical records of patients attending a tertiary referral uveitis service over a 15 year period.

Results: Of 1450 patients with uveitis, 115 (7.9\%) had neurological disease that was considered to be causally related to the eye inflammation. The most frequent neurological associations were Vogt-Koyanagi-Harada disease, primary central nervous system lymphoma, multiple sclerosis, and herpes virus infections.

Conclusions: Neurological disease is common among patients attending a uveitis service. The distinctive characteristics of the uveal inflammation may be useful in diagnosing the neurological disease.

U veitis may be associated with a variety of diseases including distinct ocular syndromes, immunologically mediated systemic diseases, infections, and masquerading syndromes such as malignancies. ${ }^{12}$ Despite recognition of a close anatomical relation, as well as immunological similarities, between the eye and the brain, the association between uveitis and neurological disease has rarely been considered. Clearly, the recognition of such associations has implications for visual and systemic prognosis and impacts patient management. We conducted a retrospective review of all patients who attended a tertiary referral uveitis service over a 15 year period to (1) establish the prevalence of concomitant neurological disease in patients with uveitis, (2) identify the neurological disorders that occur in association with uveitis, and (3) describe the types of uveitis that are associated with the most common neurological diagnoses.

\section{METHODS}

We retrospectively reviewed the medical charts of consecutive patients presenting to the tertiary care uveitis clinic at Oregon Health and Science University between September 1985 and June 2000. All cases of uveitis that were associated with neurological disease were recorded, and the specific neurological diagnosis and the form of uveitis were documented. Individuals with neurological disease that was presumed to be unrelated to their uveitis were not included. Excluded neurological conditions included those with atherosclerotic, malignant (excluding lymphoma), developmental, degenerative, and traumatic aetiologies.

Patients were evaluated under the direction of a rheumatologist. In addition to a thorough ophthalmological examination, the clinical evaluation included a complete medical history. As part of the comprehensive review of systems, all patients were questioned routinely regarding presence of the following neurological symptoms: frequent headaches, loss of consciousness, seizures, double vision, hearing loss, balance difficulty, ringing in the ears, trouble swallowing, numbness, weakness, and memory changes. General physical examination and laboratory investigations were performed as indicated by history. A more complete description of the first 236 patients in this population ${ }^{1}$ and an algorithm used to evaluate these patients ${ }^{3}$ have been published previously. Published diagnostic criteria were used to make a specific neurological diagnosis. The diagnosis of multiple sclerosis was made in consultation with a neurologist, in patients with multifocal relapsing central nervous system (CNS) disease, magnetic resonance image (MRI) abnormalities compatible with demyelination and increased cerebrospinal fluid (CSF) immunoglobulin synthesis rates. ${ }^{4}$ Diagnoses of VogtKoyanagi-Harada (VKH) disease ${ }^{5}$ and Behçet's disease ${ }^{6}$ were made on the basis of characteristic clinical features including dermatological, neurological, and ocular abnormalities. Patients with sarcoidosis had either biopsy proved noncaseating granuloma or symmetric hilar adenopathy in association with uveitis. ${ }^{7}$ Collagen vascular diseases were diagnosed by a rheumatologist on clinical signs, the presence of autoantibodies and, in the case of Sjögren's syndrome, an abnormal salivary gland biopsy. ${ }^{8}$ Cogan's syndrome was confirmed by an otolaryngologist in patients with uveitis and concurrent inflammation of the eighth cranial nerve. ${ }^{9}$ A diagnosis of CNS vasculitis was based on cerebral angiographic abnormalities. ${ }^{10}$ Primary lymphoma was diagnosed by histopathological examination of vitreous and/or brain biopsy specimens. ${ }^{11}$ Patients with neurosyphilis had neurological signs and/or CSF pleocytosis, in addition to serological evidence of active syphilis. ${ }^{12}$ In some cases of uveitis and neurological disease, a specific diagnosis could not be reached, but the two entities were believed to be associated as a result of a strong temporal relation, evidence for inflammatory aetiology of both entities, and lack of other identifiable cause for both the uveitis and the neurological disease.

\section{RESULTS}

Of 1450 patients with uveitis, $115(7.9 \%)$ had neurological disease that was judged to be causally related to the eye inflammation. A list of the neurological diagnoses is presented in table 1. Specific diseases in which uveitis and neurological pathology were most frequently associated included VKH disease $(\mathrm{n}=16)$, primary CNS lymphoma $(\mathrm{n}=16)$, multiple sclerosis $(\mathrm{n}=14)$, and herpes virus infections $(n=10)$. Twenty four patients had neurological disorders of unknown aetiology, including meningitis, myelitis and inflammation of a nerve, considered to be causally related to uveal inflammation. In VKH disease, neurological involvement was common, affecting 59\% of 29 patients. In contrast, $25 \%$ of 20 patients with ocular Behçet's disease and $7 \%$ of 74 patients with sarcoid uveitis had neurological disorders.

Of the 16 patients with VKH disease, bilateral posterior or panuveitis with serous retinal detachments was documented for nine individuals $(56 \%)$, and in six additional individuals $(38 \%)$ there was chronic bilateral uveitis with

Abbreviations: CNS, central nervous system; CSF, cerebrospinal fluid; $\mathrm{MRI}$, magnetic resonance imaging; $\mathrm{TNF}$, tumour necrosis factor; $\mathrm{VKH}$, Vogt-Koyanagi-Harada 
Table 1 Neurological diseases occurring in association with uveitis in a cohort of 1450 patients with uveitis

\begin{tabular}{ll}
\hline Neurological disease & No of patients (\%) \\
\hline Vogt-Koyanagi-Harada disease & $16(1.1)$ \\
Primary central nervous system lymphoma & $16(1.1)$ \\
Multiple sclerosis & $14(1.0)$ \\
Herpes virus infections* & $10(0.7)$ \\
Optic neuritis & $7(0.5)$ \\
Sarcoidosis & $5(0.3)$ \\
Behçet's disease & $5(0.3)$ \\
Collagen vascular disease & $5(0.3)$ \\
Syphilis & $5(0.3)$ \\
Cogan's syndrome & $3(0.2)$ \\
Central nervous system vasculitis & $2(0.1)$ \\
Othert & $4(0.3)$ \\
Unknown causeł & $24(1.7)$ \\
Total & $115(7.9)$ \\
\hline
\end{tabular}

*Neurological manifestations of herpes virus infections included encephalitis, CSF pleocytosis, cerebral infarct, optic neuritis, and other cranial neuropathies.

fOther neurological diseases included Lyme disease, Takayasu's disease, bacterial meningitis, and Bell's palsy.

‡Neurological diseases of unknown cause included meningitis, myelitis, and inflammation of a nerve.

clear abnormality of posterior pole pigmentation. Thirteen of the 16 patients $(81 \%)$ who were diagnosed with primary CNS lymphoma, had vitritis, which was bilateral in 11 cases and unilateral in two cases. Subretinal infiltrates were associated with vitritis in just four patients $(25 \%)$. Seven individuals with multiple sclerosis $(50 \%)$ had pars planitis, and bilateral granulomatous anterior uveitis was present in four other individuals (29\%). Herpetic infection was manifest as unilateral anterior uveitis with raised intraocular pressure in four patients $(40 \%)$. These cases were also characterised by keratitis and/or iris atrophy. Acute retinal necrosis was the presentation of herpetic infection in four additional patients (40\%).

\section{DISCUSSION}

Despite the relative rarity of uveitis among patients with neurological disease, many of these patients have neurological disease. In our tertiary referral uveitis practice, approximately $8 \%$ of patients had neurological disease. Our data were collected over an extended period, during which time the diagnostic criteria for several neurological diseases have been refined. Consequently, it is possible that we have underestimated the prevalence of neurological disease in uveitis, although the thorough history should have identified most neurological disorders even if a specific diagnosis could not be assigned. The most frequently encountered neurological associations of uveitis were VKH disease, primary CNS lymphoma, multiple sclerosis, and herpes virus infections. A diverse range of other neurological diseases, including infectious entities, were more rarely associated with uveitis. Approximately one in five patients had a neurological disorder that did not fit within an aetiological category. Although this study included 1450 patients with a spectrum of uveitis syndromes, we did not observe some reported neurological associations, such as acute posterior multifocal placoid pigment epitheliopathy, ${ }^{13}$ Whipple's disease, ${ }^{14}$ and HTLV-1 infection. ${ }^{15}$

A neurological diagnosis may have important implications for patient management. The outcome of two common associations, primary CNS lymphoma and herpes virus infections, may be adversely impacted by institution of immunosuppressive therapies that are appropriate for many other forms of uveitis. While the treatment of choice for VKH disease is a 6-12 month course of oral prednisone, such extended treatment with systemic corticosteroids is often avoided in other chronic forms of uveitis. Because of the potential for tumour necrosis factor (TNF) blockers to exacerbate or precipitate demyelinating disease, ${ }^{16}$ uveal inflammation associated with multiple sclerosis is a specific contraindication for these agents, which are being increasingly employed to treat uveitis. ${ }^{17}$

Our results suggest that an appreciation of uveitis and its distinctive presentations can aid in the differential diagnosis of neurological disease. In VKH disease, bilateral posterior or panuveitis complicated by serous retinal detachments was documented in over half of patients. Other patients, who presented with uveitis subsequent to the acute phase of the disease, were observed to have a bilateral abnormality of the fundus pigmentation. Multiple sclerosis was associated with pars planitis or bilateral granulomatous anterior uveitis in three quarters of affected individuals. In $80 \%$ of patients with herpes virus infection, the clinical picture was either an acute retinal necrosis or hypertensive anterior uveitis with keratitis and/or iris atrophy. The clear exception is uveitis secondary to primary CNS lymphoma, which presented less distinctive clinical features, masquerading as an immune mediated vitritis in most patients. Although the presence of subretinal infiltrates heighten the suspicion of malignancy, in our series only one quarter of patients showed this sign.

\section{Authors' affiliations}

\section{J R Smith, J T Rosenbaum, Casey Eye Institute, Oregon Health and} Science University, Portland, OR, USA

This work was supported by grants from Research to Prevent Blindness (Career Development Award to JRS and Senior Scholar Award to JTR) and the Rosenfeld Family Trust.

Correspondence to: Justine R Smith, Casey Eye Institute, Oregon Health and Science University, 3375 SW Terwilliger Blvd, Portland, OR 972394197, USA; smithjus@ohsu.edu

Accepted for publication 25 May 2004

\section{REFERENCES}

1 Rosenbaum JT. Uveitis. An internist's view. Arch Intern Med 1989;149:1173-6.

2 Rodriguez A, Calonge $M$, Pedroza-Seres $M$, et al. Referral patterns of uveitis in a tertiary eye care center. Arch Ophthalmol 1996;1 14:593-9.

3 Rosenbaum JT. An algorithm for the systemic evaluation of patients with uveitis: guidelines for the consultant. Sem Arth Rheum 1990;19:248-57.

4 Noseworthy JH, Lucchinetti C, Rodriguez M, et al. Multiple sclerosis. N Engl J Med 2000;343:938-52

5 Read RW, Holland GN, Rao NA, et al. Revised diagnostic criteria for VogtKoyanagi-Harada disease: report of an international committee on nomenclature. Am J Ophthalmol 2001;131:647-52.

6 International Study Group for Behçet's Disease. Evaluation of diagnostic ('classification') criteria in Behcet's disease-towards internationally agreed criteria. Br J Rheumatol 1992;31:299-308.

7 Winterbauer RH, Belic N, Moores KD. Clinical interpretation of bilateral hilar adenopathy. Ann Intern Med 1973;78:65-71.

8 Rosenbaum JT, Bennett RM. Chronic anterior and posterior uveitis and primary Sjögren's syndrome. Am J Ophthalmol 1987;104:346-52.

9 Chynn EW, Jakobiec FA. Cogan's syndrome: ophthalmic, audiovestibular, and systemic manifestations and therapy. Int Ophthalmol Clin 1996;36:61-72.

10 Wilson DJ, Braziel R, Rosenbaum JT. Intraocular lymphoma: immunopathologic analysis of vitreous biopsy specimens. Arch Ophthalmol 1992;110:1455-8.

11 Harris K, Tran D, Sickels W, et al. Diagnosing intracranial vasculitis: the roles of MR and angiography. Am J Neuroradiol 1994;15:317-330.

12 Passo MS, Rosenbaum JT. Ocular syphilis in patients with human immunodeficiency virus infection. Am J Ophthalmol 1988;106:1-6.

13 Comu S, Verstraeten T, Rinkoff JS, et al. Neurological manifestations of acute posterior multifocal placoid pigment epitheliopathy. Stroek 1996;27:996-1001.

14 Bodaghi B, Dauga C, Cassoux N, et al. Whipple's syndrome (uveitis, B27negative spondlyarthropathy, meningitis, and lymphadenopathy) associated with Arthrobacter sp infection. Ophthalmology 1998;105:1891-6.

15 Nakao K, Ohba N, Nakagawa M, et al. Clinical course of HTLV-1-associated uveitis. Jpn J Ophthalmol 1999:43:404-9.

16 Mohan N, Edwards ET, Cupps TR, et al. Demyelination occurring during antitumor necrosis factor alpha therapy for inflammatory arthritides. Arthritis Rheum 2001;44:2862-9.

17 Rosenbaum JT, Smith JR. Anti-TNF therapy for eye involvement in spondyloarthropathy. Clin Exp Rheumatol 2002;20:S143-5. 